\title{
Research of Fault Line Selection on Single-Phase Neutral Ineffectively Earthed Systems Based On Extended Prony Algorithm
}

\author{
Yan Li-Mei, Tang Qi-Hao \\ (Department of Electrical Engineering and Information, Northeast Petroleum University, Heilongjiang Daqing, \\ 163318, China)
}

\begin{abstract}
The Neutral indirectly grounded system has the characters of high stability and reliability so that has been applied in distribution network widely. By analyzing the principle and advantages of extended prony algorithm and researching the characteristics of steady state and transient process of single phase earth fault system, the method of using amplitude and phase of zero sequence current of the fault line and non-faulted line to determine the fault line is provided. The simulations of Matlab shows that the extended prony algorithm has a good performance on neutral indirectly grounded system.
\end{abstract}

Keywords: Extended prony algorithm; Neutral indirectly grounded system; Single-phase grounding fault ; Fault line detection; Simulation of verification

\section{Introduction}

Because the current of single phase to ground fault in the neutral ineffectively grounded system is generally not more than $10 \mathrm{~A}$, it is also called the small current grounding system. When the single-phase earth fault occurs, the three-phase voltage of the small current grounding system still meets the symmetrical relationship, so that the system can continue to supply power. Its high power supply reliability makes it widely used in the distribution network of $110 \mathrm{KV}$ and below ${ }^{[1]}$.

Because the short circuit current of the single phase grounding is so small that could not make the over current relay happen, it is difficult to realize the fault line selection of single phase to earth fault in small current grounding system. In the distribution network with $110 \mathrm{KV}$ and below, the single phase to earth fault is the most easy to occur in all fault types, accounting for about $80 \%$. So the research of single phase fault line selection in small current grounding system is of great application value ${ }^{[2]}$. So far for the single-phase fault line selection are mainly two kinds of methods, one is to judge by using single feature, including three methods that are based on zero sequence current, negative sequence current and applied of signal, none of the three methods can accurately achieve the fault line selection, so it has some limitations. The other is the combination of the above three kinds of single features to form a multiple criteria, so that greatly improve the accuracy of the line selection.

\section{Analysis of extended Prony Algorithm}

The exponential function is used as the mathematical model of the extended Prony algorithm, and the discrete form of its function is shown in the form of formula (1).

$$
\hat{x}(n) \sum_{n=1}^{p} b_{i} z_{i}^{n}, n=0,1, \cdots, N-1
$$

Among, $b_{i}=A_{i} \exp \left(j \theta_{i}\right), \quad z_{i}=\exp \left[\left(\alpha_{i}+j 2 \pi f_{i}\right) \Delta t\right]$ 。

$A_{i}$-amplitude; $\theta_{i}$-phase; $\alpha_{i}$-attenuation factor; $f_{i}$-oscillation frequency; $\Delta t$-sampling interval。

There are five steps to achieve Prony algorithm, the method is as follows,

1) The definition formula of sample function is given, as shown in the formula (1).

$$
r(i, j)=\sum_{n=p}^{N-1} x(n-j) x^{*}(n-i), i, j=0,1 \cdots, p
$$

The $R_{e}$ matrix is constructed based on the definition of sample function 
$R_{e}=\left[\begin{array}{cccc}r(1,0) & r(1,1) & \cdots & r\left(1, p_{e}\right) \\ r(2,0) & r(2,1) & \cdots & r\left(2, p_{e}\right) \\ \vdots & \vdots & \vdots & \vdots \\ r\left(p_{e}, 1\right) & r\left(p_{e}, 2\right) & \cdots & r\left(p_{e}, p_{e}\right)\end{array}\right], p_{e}>>p$

2) Using the singular value decomposition (total least squares (SVD TLS) algorithm to determine the effective rank of the matrix $p$, and the attenuation coefficient of total least squares estimate

3) Obtain the roots $z_{1}, \cdots, z_{p}$ of eigenfunction $1+\alpha_{1} z^{-1}+\cdots+\alpha_{p} z^{-p}=0$, and use the equation $\hat{x}(n)=-\sum_{n=1}^{p} \alpha_{i} \hat{x}(n-i), i=0,1, \cdots, N-1$ to get $\hat{x}(n), n=1, \cdots, N$ on the condition of $\hat{x}(0)=x(0)$.

4) Using the equation (5), the $b$ can be easily calculated

$\left[\begin{array}{cccc}1 & 1 & \cdots & 1 \\ z_{1} & z_{2} & \cdots & z_{p} \\ \vdots & \vdots & \vdots & \vdots \\ z_{1}^{N-1} & z_{2}^{N-1} & \cdots & z_{p}^{N-1}\end{array}\right]\left[\begin{array}{c}b_{1} \\ b_{2} \\ \vdots \\ b_{p}\end{array}\right]=\left[\begin{array}{c}\hat{x}(1) \\ \hat{x}(2) \\ \vdots \\ \hat{x}(p)\end{array}\right]$

5) The value of the amplitude of $A_{i}$, the phase of $\theta_{i}$, the frequency of $f_{i}$ and the attenuation factor of $\alpha_{i}$ are determined by the formula (5).

$$
\left.\begin{array}{c}
A_{i}=\left|b_{i}\right| \\
\theta_{i}=\arctan \left[\operatorname{Im}\left(b_{i}\right) / R_{e}\left(b_{i}\right)\right] / 2 \pi \Delta t \\
\alpha_{i}=\ln \left|z_{i}\right| / \Delta t \\
f_{i}=\arctan \left[\operatorname{Im}\left(z_{j}\right) / R_{e}\left(z_{j}\right)\right] / 2 \pi \Delta t
\end{array}\right\}, i=1, \cdots, p
$$

\section{Fault analysis of single phase grounding system}

\subsection{Characteristics of single phase to ground steady state}

When the system of which both the neutral point of the load and the power supply are not connected to the ground operates normally, there is a capacitance which the equivalent value equals to $C_{0}$ in each phase of the system to the ground ${ }^{[3]}$. Because each phase has a phase voltage, it will have a lead of 90 degree of current flowing into the earth, the current flowing through the three-phase capacitance to ground is zero. When the grounding fault of A phase occurs, the equivalent capacitance and voltage of this phase will be 0 , and the two phase voltage of the $\mathrm{B}, \mathrm{C}$ under normal operation will be $\sqrt{3}$ times of original ones. It is not difficult to see from Figure 1, the total capacity to the ground of the generator $C_{0 G}, C_{0 I}, C_{0 I I}$, line I, II are shown as the capacity in the figure. When the grounding fault of A phase of the line II occurs, the " $\rightarrow$ "in the figure indicates the distribution of capacitive current.

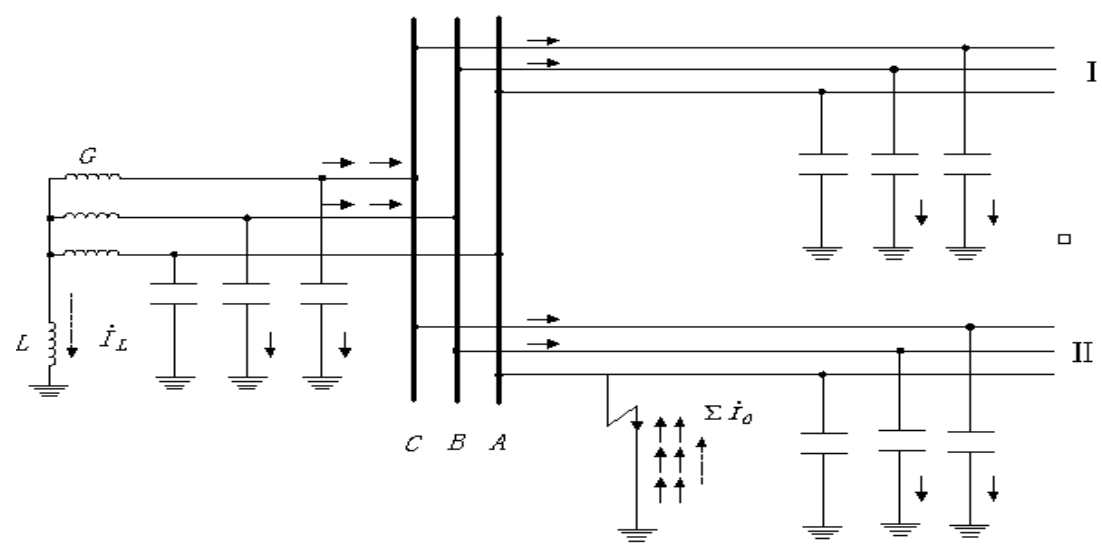

Fig.1 System of single-phase grounding current distribution 
As shown in Figure 1, there is no ground fault in line I, the grounding fault of the A phase of line II occurs, the capacitor current and effective values of the two phases of the B, C are shown respectively in the formula (6) and (7).

$$
\begin{aligned}
& \dot{I}_{k}=\left(\dot{I}_{B I}+\dot{I}_{C I}\right)+\left(\dot{I}_{B I I}+\dot{I}_{C I I}\right)+\left(\dot{I}_{B G}+\dot{I}_{C G}\right) \\
& I_{k}=3 U_{\varphi} \omega\left(C_{0 I}+C_{0 I I}+C_{0 G}\right)=3 U_{\varphi} \omega C_{0 \Sigma}
\end{aligned}
$$

The current also from A phase of line II flows back into the system, so the current out of A phase is its negative, the value and its effective value of the zero sequence current flowing through the beginning of fault lines II are shown in the formula (8) and (9):

$$
\begin{aligned}
& 3 \dot{I}_{0 I I}=\dot{I}_{A I I}+\dot{I}_{B I I}+\dot{I}_{C I I}=-\left(\dot{I}_{B I}+\dot{I}_{C I}+\dot{I}_{B G}+\dot{I}_{C G}\right) \\
& 3 I_{0 I I}=3 U_{\varphi} \omega\left(C_{0 \Sigma}-C_{0 I I}\right)
\end{aligned}
$$

Through the above analysis, we can conclude that zero sequence current of fault line in neutral point non grounded system is the sum of the non-fault component of the whole system to the ground capacitance current, the capacitive reactive power of the grounding fault line is from the line to the bus, and the non-fault line is from the bus to the line $\mathrm{e}^{[4]}$. When the circuit is grounded by arc suppression coil, the distribution of system current in single phase to ground fault is much more different from that of the neutral point grounded system.

\subsection{Characteristics of single phase grounding transient process}

Insulation breakdown is the main reason for the occurrence of single phase fault in power grid system ${ }^{[5]}$. The equivalent circuit of the transition process of the neutral point non grounded system is shown in Figure 2.By ignoring $L_{k}$ the above circuit is an $\mathrm{R}, \mathrm{L}, \mathrm{C}$ series circuit, which can be seen from Figure 2 . The transient capacitance current and zero sequence voltage $u(t)$ transition process are analyzed by this circuit. The variation of the current in the fault point is changed with the change of network parameter $\mathrm{R}, \mathrm{L}$ and $\mathrm{C}$. When $R<2 \sqrt{L / C}$, the current transition process is periodic decay, when $R>2 \sqrt{L / C}$, the current through the non-periodic decay and tend to steady state values ${ }^{[6]}$. By the analysis, the main characteristic of the zero sequence current of the small current grounding system is embodied in the fundamental frequency and the free oscillation frequency of the current ${ }^{[7]}$. The extended Prony algorithm can effectively decompose the sine function of the small current free oscillation frequency, which is very beneficial to the fault determination and selection.

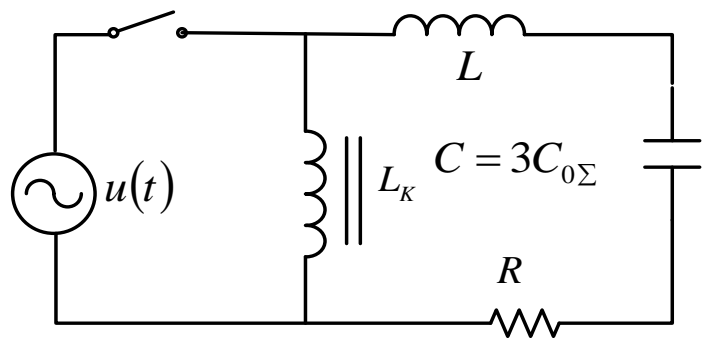

Fig. 2 equivalent network of the transition process

\section{The MATLAB simulation analysis}

Use the Matlab to establish the simulation model, the Parameters Line Distributed model is used as the line model, bus bar leads to the 5 voltage of the outlet power supply $10 \mathrm{KV}^{[8]}$. Line length are $L_{1}=18 \mathrm{~km}, L_{1}=18 \mathrm{~km}, L_{3}=10 \mathrm{~km} 7.7512 \times 10^{-9} \mathrm{~F} / \mathrm{km}, L_{4}=16 \mathrm{~km}$ and $L_{5}=6 \mathrm{~km}$. The positive line resistance, inductance and capacitance of the positive sequence are $0.17 \Omega / \mathrm{km}, 1.1714 \times 10^{-3} \mathrm{H} / \mathrm{km}$ and $12.7 \times 10^{-9} \mathrm{~F} / \mathrm{km}$, the zero sequence resistance, zero sequence inductance and zero sequence capacitance value are $0.228 \Omega / \mathrm{km}$, and $3.0956 \times 10^{-3} \mathrm{H} / \mathrm{km}$

When the transition resistance is $0 \Omega$, the end of the line 1 occurs A phase to ground fault when the phase voltage is the maximum value. It can be seen from the Figure 3 and Table 1, the zero sequence current and the amplitude of the free oscillation component of the fault line reached the maximum value, its phase with the non-fault line is about 180 degrees. Therefore, the fundamental frequency of zero sequence current and the phase and the amplitude of free oscillation can be extracted, the phase and other lines with a difference of more than 90 degrees of the line is a fault line. 

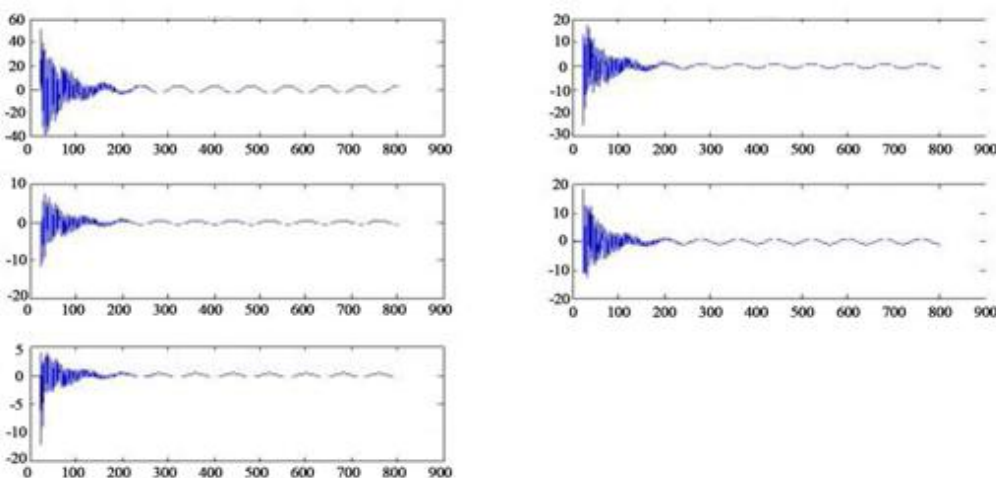

Fig.3 The waveform of each circuit when the neutral point is not grounded and the grounding resistance is $0 \Omega$

Table 1 the neutral point is not grounded, and the grounding resistance is $0 \Omega$.

\begin{tabular}{|c|c|c|c|c|c|c|}
\hline & & Line 1 & Line 2 & Line 3 & Line 4 & Line 5 \\
\hline \multirow[t]{3}{*}{ Free oscillation } & (ampere /A) & 45.1 & 17.9 & 8.3 & 14.2 & 4.7 \\
\hline & $($ hertz $/ \mathrm{Hz})$ & 744.6 & 744.6 & 744.5 & 744.5 & 744.5 \\
\hline & phase $\quad\left(\right.$ degree $\left./ /^{\circ}\right)$ & 0.4 & -179.8 & -179.8 & -179.5 & -179.3 \\
\hline \multirow{3}{*}{$\begin{array}{l}\text { fundamental } \\
\text { frequency }\end{array}$} & amplitude (ampere /A) & 2.9 & 1.2 & 0.6 & 0.8 & 0.4 \\
\hline & (hertz /Hz) & 50.0 & 50.0 & 50.1 & 49.9 & 50.0 \\
\hline & phase $\quad\left(\right.$ degree $\left./{ }^{\circ}\right)$ & 176.2 & -3.5 & -5.0 & -3.3 & -5.1 \\
\hline
\end{tabular}

When the numerical value of the transition resistance is increased, the free oscillation component will not show obvious characteristics. Figure 4 and Table 2 show that the amplitude of the access resistance increased to $200 \Omega$, the extended prony algorithm can still guarantee the accuracy of the zero sequence current of fundamental frequency component amplitude and phase, if it continues to increase the access resistance, the fundamental characteristics can only be used for the line selection.
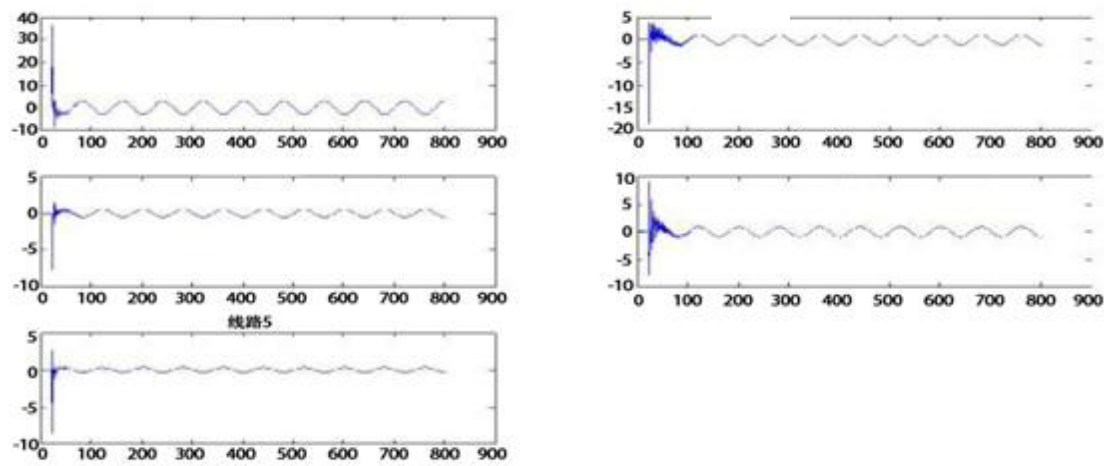

Fig.4 The waveform of each circuit when the neutral point is not grounded and the grounding resistance is $100 \Omega$

Table 2 the neutral point is not grounded, and the grounding resistance is $100 \Omega$

\begin{tabular}{|c|c|c|c|c|c|c|}
\hline & & Line 1 & Line 2 & Line 3 & Line 4 & Line 5 \\
\hline \multirow[t]{3}{*}{ Free oscillation } & amplitude (ampere /A) & 51.8 & 17.7 & 18.0 & 13.2 & 3.7 \\
\hline & frequency $\quad($ hertz $/ \mathrm{Hz})$ & 690.6 & 708.3 & 720.8 & 709.2 & 665.0 \\
\hline & phase $\left(\right.$ degree $\left./{ }^{\circ}\right)$ & 7.3 & 179.1 & 158.3 & -179.8 & -173.1 \\
\hline \multirow{3}{*}{$\begin{array}{l}\text { fundamental } \\
\text { frequency }\end{array}$} & amplitude (ampere /A) & 2.9 & 1.1 & 0.6 & 0.9 & 0.3 \\
\hline & (hertz /Hz) & 50.0 & 50.0 & 50.0 & 50.0 & 50.0 \\
\hline & phase $\left(\right.$ degree $\left./{ }^{\circ}\right)$ & 173.1 & -6.5 & -7.0 & -6.6 & -7.3 \\
\hline
\end{tabular}

\section{Conclusion}

Trough the detailed analysis of the characteristics of faults, the result that small current grounding system can still be operated for a period of time when a single phase to ground fault occurs can be got and provides the feasibility for the application of the extended prony algorithm. The extended prony algorithm can be used to extract the features of free oscillation and fundamental frequency components accurately, and to a certain extent, it can improve the ability of anti-interference and anti-noise. The simulation results show that the extended prony algorithm can select the line with single phase to ground fault accurately. 


\section{References}

[1]. CUI Xiaoping. "Studying on the Oxidization-reduction Method of Decontaminating Irritant CS", Science Technology and Engineering, 2012, vol. 12, no. 28, pp.7402-7407.

[2]. Nam, Soon Ryul, et al. "Single line-to-ground fault location based on unsynchronized phasors in automated ungrounded distribution systems", Electric Power Systems Research, 2012, vol. 86, pp.151-157.

[3]. REN Jianwen, SUN Wenwu, ZHOU Ming, et al. "Transient Algorithm for Single-line to Ground Fault Selection in Distribution Networks Based on Mathematical Morphology", Automation of Electric Power Systems, 2008, vol. 32, no. 1, pp.70-75

[4]. YIN Dechang, ZHAO Feng. "Simulation Research and Fault Line Selection of Single-phase Grounding Fault in 10kV Distribution Network", Science Technology and Engineering, 2013, vol. 13, no. 6, pp.1609-1616.

[5]. Tan, Junyuan. "The Line-to-Ground Fault and Fault Location Analysis in Distribution Networks", Electrical Engineering, 2008 , vol. 10, pp.66-69.

[6]. Zhang, Xin Hui, et al. "Analysis of transient dominant frequency signal for single-phase earthed fault based on prony algorithm" China International Conference on Electricity Distribution IEEE, 2008, pp.1-6.

[7]. Yuanyuan, W., Z. Xiangjun, and S. Sheng. "Grounding Fault Protection with Phase Current Difference for Ineffectively Earthed Power Systems." 2006, vol. 5, pp.2645-2650.

[8]. Zhong Wei-Zhi, Zhong Dan-Dan, Jing Qing-Feng, et al. "Flat fast-fading channel prediction algorithm on the basis of extended Prony algorithm", Journal of South China University of Technology (Natural Science), 2015, vol. 43, no. 3, pp.78-83. 